Relations industrielles

Industrial Relations

\title{
Index Volume 14 - 1959
}

Volume 14, numéro 4, octobre 1959

URI : https://id.erudit.org/iderudit/1022168ar

DOI : https://doi.org/10.7202/1022168ar

Aller au sommaire du numéro

Éditeur(s)

Département des relations industrielles de l’Université Laval

ISSN

0034-379X (imprimé)

1703-8138 (numérique)

Découvrir la revue

Citer ce document

(1959). Index Volume 14 - 1959. Relations industrielles / Industrial Relations,

14(4), 634-639. https://doi.org/10.7202/1022168ar

Tous droits réservés (C Département des relations industrielles de l’Université Laval, 1959
Ce document est protégé par la loi sur le droit d'auteur. L’utilisation des services d'Érudit (y compris la reproduction) est assujettie à sa politique d'utilisation que vous pouvez consulter en ligne.

https://apropos.erudit.org/fr/usagers/politique-dutilisation/ 


\section{IN D EX VOLUME 14 - 1959}

Bargaining: A Closer Look, Variations from Pattern, Kenneth Alexander, (no 2), pp. 211-230.

Bénéfices au Canada, La participation aux, Gaston Cholette, (no 4), pp. 553-570.

Bénéfices aux Etats-Unis, Les syndicats ouvriers et la participation aux, Gaston Cholette, (no 2), pp. 200-208.

Bénéfices aux Etats-Unis, La participation, Gaston Cholette, (no 1), pp. 2-34.

Benefut, Management Attitudes Towards Fringe, T.H. Robinson, (no 4), pp. 505517.

Caisses de retraite - Nouvelle source de capital, Les, J. Alasco, (no 3), pp. 404409.

Canada, La participation aux bénéfices au, Caston Cholette (no 4), pp. 553-570.

Canada, Le respect des charges de familles dans la taxation et la sécurité sociale au, Claude Morin, (no 4), pp. 589-597.

Canada, Profit Sharing In, Gaston Cholette, (no 4), 571-572.

Canadienne, Les problèmes constants de la politique économique, Claude Morin, (no 3), pp. 410-413.

CCCL, to the Quebec Government, Unemployment - Brief submitted by the AFL and the, (no 1), pp. 118-125.

Chômage, Nos responsabilités chrétiennes en face du, Cardinal Paul-Emile Léger, (no 1), pp. 126-139.

Chômage - Mémoire conjoint FTQ-CTCC au gouvernement de la province de Québec, Le, (no 1), pp. 110-117.

Collective, Les employés de bureau et la négociation, Léo Roback, (no 1), pp. 6874.

Commerce du Canada, la liberté d'entreprise et les relations du travail, La Chambre de, (no 2), pp. 298-301.

Confessionnalite, La CTCC, laction politique et la, (no 4), pp. 604-605.

Conventions-clés, Résumé: Etude des difficultés d'adaptation aux, Kenneth Alexander, (no 2), pp. 23l-232.

Corporation? Les épiciers doivent-ils former une, Emile Gosselin, Charles Lemelin, (no 1), pp. 59-67.

CTC-PSD d Winnipeg, Le colloque, Fernand Bourret (no 4), pp. 573-588. 
CTC, Mémoire au Gouvernement fédéral: Législation du travail, (no 3), pp. 432435.

CTCC au gouvernement de la province de Québec, le chômage - Mémoire conjoint FTQ- (no 1), pp. 110-117.

CTCC, l'action politique et la confessionnalité, La, (no 4), pp. 604.

CTCC, Mémoire au Gouvernement fédéral: Le boycottage secondaire; Les briseurs de grèves; Luttes au syndicalisme. (no 3), pp. 430-432.

Droits civils dans le fonctionnarisme, Déclaration de principes sur les, American Civil Liberties, (no 4), pp. 598-603.

Droit du travail, Le, Marie-Louis Beaulieu, (no 3), pp. 414-418.

Economique canadienne, Les problèmes constants de la politique, Claude Morin, (no 3), pp. 410-413.

Economique ou récession? Prospérité, Claude Morin, (no 1), pp. 85-88.

Entreprise et les relations du travail, La Chambre de Commerce du Canada, la liberté d', (no 2), pp. 298-301.

Entreprise, dans les petites et moyennes entreprises, La succession du Chef $d$, S. Cambien, (no 2), pp. 246-264.

Epiciers doivent-ils former une corporation? Les, Emile Gosselin, Charles Lemelin, (no 1), pp. 59-67.

Etats-Unis, La participation aux bénéfices aux, Gaston Cholette (no 1), pp. 2-34.

Etats-Unis, Résumé: Vers une théorie du développement des unions ouvrières, Joseph L. Massie, (no 1), pp. 48-50.

Etats-Unis, Les syndicats ouvriers et la participation aux bénéfices aux, Gaston Cholette, (no 2), pp. 200-208.

Federal Structures and Social Legislation, Summary: René H. Mankiewicz, (no 3), pp. 377-378.

Fédérales et la législation sociale, Les structures, René H. Mankiewicz, (no 3), pp. 359-376.

Fonctionnarisme, Déclaration de principes sur les droits civils dans le, American Civil Liberties, (no 4), pp. 598-603.

Formation de personnel et leur évaluation, Organisation des programmes de, Jacques Lucier, (no 2), pp. 232-244.

FTQ-CTCC au gouvernement de la province de Québec, Le chômage - Mémoire conjoint, ( no 1), pp. 110-117.

F.T.Q. (Québec 1958), Le 2è congrès de la, Guy Lamarche, (no 1), pp. 51-58.

Grève des réalisateurs de Radio-Canada, La, Michel Roy, (no 2), pp. 265-276.

Incentive Systems and Critics, Summary: Wage-, Jean-Paul Deschênes, (no 2), pp. 190-199. 
Incentive Systems: A Critic (II), Summary: Wage-, Jean-Paul Deschênes, (no 3), pp. 331-336.

Incentive Systems: A Critic (III): The Human Factor, Wage-, Jean-Paul Deschênes, (no 4), pp. 474-484.

Labor Attitudes on Profit Sharing in the United States, Summary: Gaston Cholette, (no 2), pp. 209-210.

Labor-Management Relations 1958-1959, The State of, Arthur Goldberg, (no 1), pp. 75-84.

Labor Movements, Comparative Aspects of, Rodney F. White, (no 3), pp. 337354.

Legislation, Summary: Federal Structures and Social, René H. Mankiewicz, (no 3), pp. 377-378.

Legislation sociale, Les structures fédérales et la, René H. Hankiewicz, (no 3), pp. 359-376.

Management Attitudes Towards Fringe Benefits, T.H. Robinson (no 4), 505-516.

Management Relations 1958-1959, The State of Labor-, Arthur Goldberg, (no 1), pp. $75-84$.

Mouvements ouvriers, Résumé: Aspects comparatifs des, Rodney F. White (no 3), pp. $355-358$.

Négociation collective, Les employés de bureau et la, Léo Roback, (no 1), pp. 6874.

Ontario, Le rapport de la commission d'enquête sur les relations du travail de la Législature de l'. Gaston Cholette, (no 3), pp. 379-403.

Ouvriers, Résumé: Aspects comparatifs des mouvements, Rodney F. White, pp. 355358.

Pattern Bargaining: A Closer Look, Variations from, Kenneth Alexander, (no 2), pp. 211-230.

Personnel et leur évaluation, Organisation des programmes de formation de, Jacques Lucier, (no 2), pp. 232-244.

Personnel Training Programs: Organization and Evaluation, Summary: Jacques Lucier, (no 2), pp. 245-246.

Police et les différends du travail, La, (no 3), pp. 427-429.

Politique économique canadienne, Les problèmes constants de la, Claude Morin, (no 3), pp. 410-413.

Politique et la confessionnalité, La CTCC, l’action, (no 4), pp. 604.

Politique, Syndicalisme ouvrier et action, (no 1), pp. 154-158.

Procédures: résultats d'une expérience, Analyse des systèmes et, Roger Gosselin, (no 4), pp. 485-502. 
Profut Sharing in the United States of America, Summary: Gaston Cholette, (no 1), pp. 35-37.

Profit Sharing in the United States, Summary: Labor Attitudes on, Gaston Cholette, (no 2), pp. 209-210.

Profit Sharing in Canada, Gaston Cholette, (no 4), pp. 571-572.

PSD à Winnipeg, Le colloque CTC-, Fernand Bourret, (no 4), pp. 573-588.

OFL and the CCCL to the Quebec Government, Unemployment - Brief submitted by the, (no 1), pp. 118-125.

Québec, Le chômage - Mémoire conjoint FTQ-CTCC au gouvernement de la province de, (no 1), pp. 110-117.

Radio-Canada, La grève des réalisateurs de, Michel Roy, (no 2), pp. 265-276.

Recession? Prospérité économique ou, Claude Morin, (no 1), pp. 85-88.

Relations du travail de la Législature de l'Ontario, Le rapport de la commission d'enquête sur les, Gaston Cholette (no 3), pp. 379-403.

Relations du travail, La Chambre de Commerce du Canada, La liberté d'entreprise et les, (no 2), pp. 298-301.

Rémunération selon le rendement, Une critique de la, Jean-Paul Deschênes, (no 2), pp. 178-189.

Rémunération selon le rendement (II), Line critique de la, Jean-Paul Deschênes, (no 3), pp. 322-330.

Rémunération selon le rendement (III), le facteur humain, Une critique de la, Jean-Paul Descênes, (no 4), pp. 458-473.

Retraite - Nouvelle source de capital, Les caisses de, J. Alasco, (no 3), pp. 404-409.

Sécurité sociale au Canada, Le respect des charges de familles dans la taxation et la, Claude Morin (no 4), pp. 589-597.

Sécurité sociale et la dette publique, La, Claude Morin (no 2), pp. 277-279.

Syndicalisme ouvrier et action politique, (no 1), pp. 154-158.

Syndicats ouvriers et la participation aux bénéfices aux Etats-Unis, Les, Gaston Cholette, (no 2), pp. 200-208.

Systèmes de procédures: résultats d'une expérience, Analyse des, Roger Gosselin (no 4), 485-502.

Systems and Procedures, Analysis of, Roger Gosselin (no 4), pp. 503-504.

Taxation et la sécurité sociale au Canada, Le respect des charges de famille dans la, Claude Morin, (no 4), 589-597.

Training Programs: Organization and Evaluation, Summary: Personnel, Jacques Lucier, (no 2), pp. 245-246.

Travail, Le droit du, Marie-Louis Beaulieu, (no 3), pp. 414-418.

Travail de la Législature de l'Ontario, Le rapport de la commission d'enquête sur les relations du, Gaston Cholette, (no 3), pp. 379-403. 
Travail, La police et les différends du, (no 3), pp. 427-429.

UAW Experience, Impartial Umpireships: The General Motors, Gabriel N. Alexander, (no 4), pp. 517-552.

Unemployment and our Responsibilities as Christians, Cardinal Paul-Emile Léger, (no 1), pp. 126-139.

Unemployment - Brief submitted by the AFL and the CCCL to the Quebec Government, (no 1), pp. 118-125.

Unionism in the U.S., Toward a Theory of the Development of, Joseph L. Massie, (no 1), pp. 38-47.

Unions ouvrières aux Etats-Unis, Résumé: Vers une théorie du développement des, Joseph L. Massie (no 1), pp. 48-50.

United States, Summary: Labor Attitudes on Profit Sharing in the, Gaston Cholette, (no 2), pp. 209-210.

U.S. Toward a Theory of the Development of Unionism in, Joseph L. Massie (no 1), pp. 38-47.

Wage-Incentive Systems and Critics, Summary: Jean-Paul Deschênes, (no 2), pp. 190-199.

Wage-Incentive Systems: A Critic (II), Summary: Jean-Paul Deschênes, (no 3), pp. 331-336.

Wage-Incentive Systems: A Critic (III): The Human Factor, Jean-Paul Deschênes, (no 4), pp. 474-484.

\section{JURISPRUDENCE DU TRAVAIL}

Ancienneté: Promotion interdépartementale et, Emile Gosselin (no 1), pp. 89-91.

Une nouvelle convention annulle-t-elle des conventions antérieures?, Grief, (no 3), pp. 419-426.

Arbitrale de la convention collective, L'interprétation, Marie-Louis Beaulieu, (no 1), p. 101.

Civil Code, The Criminal Law and the, Erskine Buchanan, Philip Cutler, Paul E. Renault, (no 1), pp. 102-109.

Convention écrite, Opposition entre usage et, Roger Chartier, (no 1), pp. 92-100.

Convention annuelle-t-elle les conventions antérieures, Grief d'ancienneté: Une nouvelle, (no 3), pp. 419-426.

Grief, Comment doivent se calculer les délais pour loger un, (no 4), pp. 605-613. Managements Rights, Lay-off, Seniority Clause and, (no 4), pp. 614-616.

Rand dans la Province de Québec, Légalité de la formule, (no 2), pp. 280-293.

« Routine », Changement de, Léo Roback, (no 2), pp. 294-297.

Seniority Clause and Managements Rights, Lay-off, (no 4), pp. 614-616. 


\section{RECENSION}

Barbash, Jack, The Practice of Unionism, (no 4), p. 623. Barnes, Ralph M., Motion and Time Study, (No 1), p. 164. Blanc-Jouvan, Xavier, Les rapports collectifs de travail aux Etats-Unis (no 1), pp. 160-161. Brown, Douglas J. and Harbison, Frederick, High-Talent Manpower for Sciences and Industry, (no 3), pp. 446-448. Bureau International du Travail, Introduction à l'Etude du travail, (no 4), p. 626. Bureau International du Travail, Le coût de la Sécurité sociale, 1949-1954, (no 4), p. 619. Bureau International du Travail, Le coût des soins médicaux, (no 4), p. 620 . Centre d'études des problemes industriels, Forces et faiblesses des entreprises familiales, (no 3), pp. 444-445. Conférence internationale du Travail, Genève, Les problèmes des travailleurs non manuels, y compris le personnel technique, les cadres, etc., (no 3), pp. 435-437. Cornell University, American Labor's Role in Less Developped Countries, (no 4), p. 621. Cronin, Rev. John F., Social Principles and Economic Life, (no 4), p. 622. Daniels, Walter M., The American Labor Movement, (no 4), p. 621. Danty-Lafrance, Louis, Danty-Lafrance, Jean, Pratique de la rémunération du travail: Tout ce qu'il faut savoir sur les salaires (no 3), pp. 445446. Davis, Paul T. and Pollock, Ross, Executives for Government: Central Issues of Federal Personnel Administration, (no 2), pp. 308-310.Ehrmann, Henry W., Organized Business in France (no 2), pp. 302-303, Ehrmann, Henry W., La politique du Patronat français 1936-1955, (no 4), p. 627. Fear, R.A., The Evaluation Interview, (no 2), p. 304. Giner, Carlos et Dranzadi, Dionisio, En la Escuela de lo social, (no 3), p. 440. Hamelin, A., Les doctrines économiques, (no 3), p. 444. Hardy, Ls-Laurent, Brève Histoire du Syndicalisme ouvrier au Canada, (no 2), p. 303. Haro, A.E.C., The Principle of Industrial Relations (no 4), p. 622. Industrial and Labor Relations Review, The Taft-Hartley Act After Ten Years: A symposium, (no 1), pp. 161-164. International Labour Office, Labour Cost in European Industry, (no 4), p. 624. Karsh, Bernard, Diary of a Strike, (no 3), pp. 438-439. Landsberger, Henry A., Hawthorne Revisited: Management and the Worker, its Critics, and Development in Human Relations in Industry, (no 2), p. 306. Maier, N.F.R., The Appraisal Interview, (no 2), p. 303. Mireault, Réal, L'ancienneté dans les promotions, (no 1), p. 159. Morris, James O., Conflict within the AFL, (no 4), p. 625. Perlman, Mark, Judges in Industry, A Study of Labour Arbitration in Australia, (no 3), pp. 437-438. Rayback, Joseph G., A History of American Labour, (no 4), p. 625. Reynolds, L., Gand Taft, C.H., The Evolution of Wage Structure, (no 2), p. 310. Roberts, B.C., Union in America, (no 4), p 617. Rollet, Henri, L'Action sociale des catholiques en France, (1871-1914), (no 3), pp. 439-440. Rustant, M. L'Automation, ses conséquences humaines et sociales, (no 3), p. 449. Sartain, A.Q., North. A.J., Strange, J.R., Chapman, H.M., Understanding Human Behavior, (no 1), p. 164. Sturmthal, Adolf, Contemporary Collective Bargaining in Seven Countries, (no 3), pp. 440-444. Tannenbaum, Arnold S. et Kahn, Robert L., Participation in Union Locals, (no 2), p. 305. 